\title{
Angular correlations in breakup of three-body halo nuclei
}

\author{
E. Garrido \\ Instituto de Estructura de la Materia, CSIC, Serrano 123, E-28006 Madrid, Spain \\ D. V. Fedorov and A. S. Jensen \\ Institute of Physics and Astronomy, Aarhus University, DK-8000 Aarhus C, Denmark
}

(Received 4 June 1998)

\begin{abstract}
We use the three-body model and the sudden approximation to compute angular correlations in high-energy fragmentation reactions of two-neutron halos on light targets. The contribution from one-neutron absorption by far dominates over that of neutron scattering. We use ${ }^{6} \mathrm{He}(n+n+\alpha)$ and ${ }^{11} \mathrm{Li}\left(n+n+{ }^{9} \mathrm{Li}\right)$ as examples and study the dependence of the predictions of this model on different physical assumptions and parameters. [S0556-2813(98)50111-2]
\end{abstract}

PACS number(s): 25.60.-t, 25.60.Gc, 21.45.+v

Introduction. A new class of nuclear states, called halos, was discovered about ten years ago [1]. Halos are spatially extended states with small one or two-nucleon separation energies and low orbital angular momenta. The systems can be understood essentially as few-body systems where we can separate the coordinates into tightly bound intrinsic (core) and loosely bound external (halo) degrees of freedom. The characteristic properties, unusual in the nuclear context, are related to the few halo degrees of freedom while the core can be assumed to be inert. Prominent examples are the twoneutron halos consisting of two neutrons and a core. The properties of halo systems have been remarkably well described by three-body models [1-5]. The lesson is clearly that properties related to the halo degrees of freedom (the core remains intact) can be deduced from three-body models.

However, the recently measured neutron angular correlations in fragmentation reactions of Borromean two-neutron halos are apparently sensitive to both the reaction mechanism and the halo structure [6]. A satisfactory description is not available. Other previously measured neutron momentum distributions in high-energy breakup reactions have been reproduced in a three-body model where the sudden approximation and the final state interactions are decisive ingredients $[3,4]$. Recently this model was extended to include both absorption and diffraction of the halo particles on the target and, in addition, the absolute two-neutron removal cross sections were successfully calculated [5]. The purpose of this Rapid Communication is to analyze the recently measured neutron angular correlations within this new model. We shall study the dependence on various physical parameters, compare to available data, see how far the model can go, possibly suggest new reaction mechanisms, and predict yet unobserved neutron correlations.

Model and method. The spatially extended three-body halo collides with a relatively small target at high energy. Then the probability that more than one of the constituents interacts strongly with the target is very small. The differential cross section $d \sigma$ is then to a good approximation a sum of three terms $d \sigma^{(i)}$ each describing the independent contribution to the process from the interaction between the target and the halo particle $i$. This is the assumptions used in the classical formulation for a weakly bound projectile [7]. We neglect the binding energy of the initial three-body bound state compared to the high energy of the beam. The reaction is then described as three particles independently interacting with the target as if each particle was free.

The process is described as removal of one particle (participant) while the other two particles (spectators) both survive undisturbed. The participant is either absorbed or elastically scattered by the target. The final state then consists of two independent subsystems, i.e., the two spectators and the target plus participant. The final state interaction between spectators is necessarily the same as in the initial three-body bound state which is described in agreement with available experimental information $[3,4]$. Since we only need to account for details of the scattered particle we employ a phenomenological optical potential where all other processes are included as absorption from the elastic channel [8].

The coordinates, $\mathbf{r}_{j k}, \mathbf{R}$, and $\mathbf{R}^{\prime}$, used to describe the reaction are sketched in Fig. 1. We denote the conjugate momenta by the corresponding $\mathbf{p}$ and use primes for the final states. We neglect the Coulomb interaction and assume that the target has zero spin. With the assumptions of two independent subsystems in the final state, we must find both elastic and absorption halo-target differential cross sections as products [5] of participant-target (elastic or absorption) cross sections, and the averaged spectator overlap matrix element [3], i.e.,

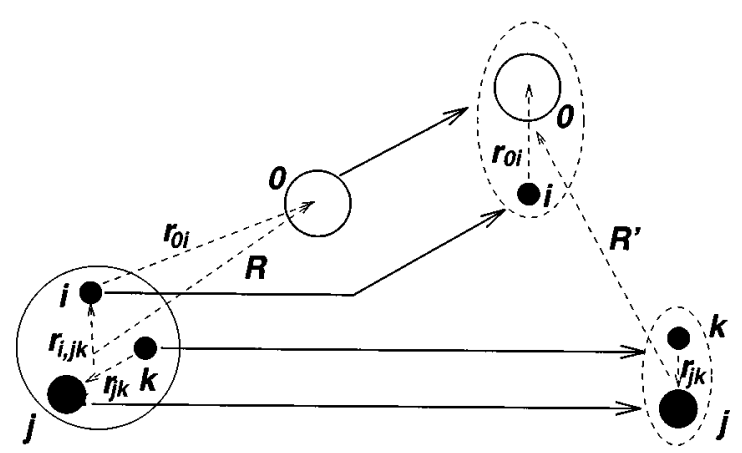

FIG. 1. Sketch of the reaction and the coordinates used. The target is labeled by 0 , and $\{i, j, k\}$ label the particles within the three-body projectile. 


$$
\begin{aligned}
& \frac{d^{9} \boldsymbol{\sigma}_{e l}^{(i)}\left(\mathbf{P}^{\prime}, \mathbf{p}_{j k}^{\prime}, \mathbf{p}_{0 i}^{\prime}\right)}{d \mathbf{P}^{\prime} d \mathbf{p}_{j k}^{\prime} d \mathbf{p}_{0 i}^{\prime}} \\
& \quad=\frac{d^{3} \boldsymbol{\sigma}_{e l}^{(0 i)}\left(\mathbf{p}_{0 i} \rightarrow \mathbf{p}_{0 i}^{\prime}\right)}{d \mathbf{p}_{0 i}^{\prime}} \frac{1}{2 J+1} \sum_{M s_{j k} \Sigma_{j k} \Sigma_{i}}\left|M_{s_{j k} \Sigma_{j k} \Sigma_{i}}^{J M}\right|^{2},
\end{aligned}
$$

$$
\frac{d^{6} \sigma_{a b s}^{(i)}\left(\mathbf{P}^{\prime}, \mathbf{p}_{j k}^{\prime}\right)}{d \mathbf{P}^{\prime} d \mathbf{p}_{j k}^{\prime}}=\sigma_{a b s}^{(0 i)}\left(p_{0 i}\right) \frac{1}{2 J+1} \sum_{M s_{j k} \Sigma_{j k} \Sigma_{i}}\left|M_{s_{j k} \Sigma_{j k} \Sigma_{i}}^{J M}\right|^{2},
$$

where $d^{3} \sigma_{e l}^{(0 i)}\left(\mathbf{p}_{0 i} \rightarrow \mathbf{p}_{0 i}^{\prime}\right) / d \mathbf{p}_{0 i}^{\prime}$ is the participant-target differential elastic cross section, $\sigma_{a b s}^{(0 i)}$ is the participant-target absorption cross section, $\Sigma_{i}$ and $\Sigma_{i}^{\prime}$ are spin projections of halo particle $i$ before and after the reaction, $s_{j k}$ and $\Sigma_{j k}=\Sigma_{j}$ $+\Sigma_{k}$ are total spin and projection of the halo particles $j$ and $k, M_{s_{j k} \Sigma_{j k}, \Sigma_{i}}$ is the overlap matrix element between initial and final states of the spectator wave functions [3], and $J$ and $M$ are total angular momentum and projection on the beam direction of the initial halo wave function.

In the experiment the core and a neutron are detected in coincidence with velocities approximately equal to the beam velocity. Thus in agreement with the participant-spectators approximation one neutron reacts with the target without destroying or significantly affecting the motion of the core and the other neutron. The finite extension of the projectile and the target therefore requires that only configurations where the participant is sufficiently far away from the spectators can contribute to the reaction. We account for this by omitting those geometric configurations in the initial wave function where the participant $(n)$ is closer to the two spectators ( $n$ and $c$ ) than cut off distances $r_{n n}$ and $r_{n c}$, which are treated as parameters. This shadowing effect substantially reduces the absolute values of the cross sections $[5,9]$.

Observable and parameters. Recently a new observable was measured after fragmentation of a two-neutron halo nucleus on a carbon target. The projectile interacts with the target and the angular distribution of the relative momentum of the detected neutron-core system is measured in a coordinate system with the $z$ axis along its center of mass momentum [6]. This process has a contribution of about $70 \%$ from absorption of the participant neutron [10]. The remaining about 30\% arises from elastic scattering of the participant neutron, which leaves two neutrons in the final state. Subsequently the equal contributions $(\approx 15 \%)$ from the spectator neutron (absorption-like distribution) or the participant neutron are measured. The contribution from the interaction of the core with the target is expected to be negligibly small [5].

We shall first concentrate on the $\approx 85 \%$ of the absorptionlike distribution. Then the relative momentum is $\mathbf{p}_{j k}^{\prime}$ and the center-of-mass momentum is the conjugate final state momentum $\mathbf{p}_{i, j k}^{\prime}$ of the coordinate $\mathbf{r}_{i, j k}$, see Fig. 1. The angular correlation is then computed from Eq. (2) by integration over all momentum coordinates except the angle $\theta$ between these $\mathbf{p}_{j k}^{\prime}$ and $\mathbf{p}_{i, j k}^{\prime}$.

We consider the nuclei ${ }^{6} \mathrm{He}(n+n+\alpha)$ and ${ }^{11} \mathrm{Li}(n+n$ $+{ }^{9} \mathrm{Li}$ ) with the wave function obtained by solving the Faddeev equations in coordinate space [3] with the potentials from [11]. The resulting three-body wave functions have

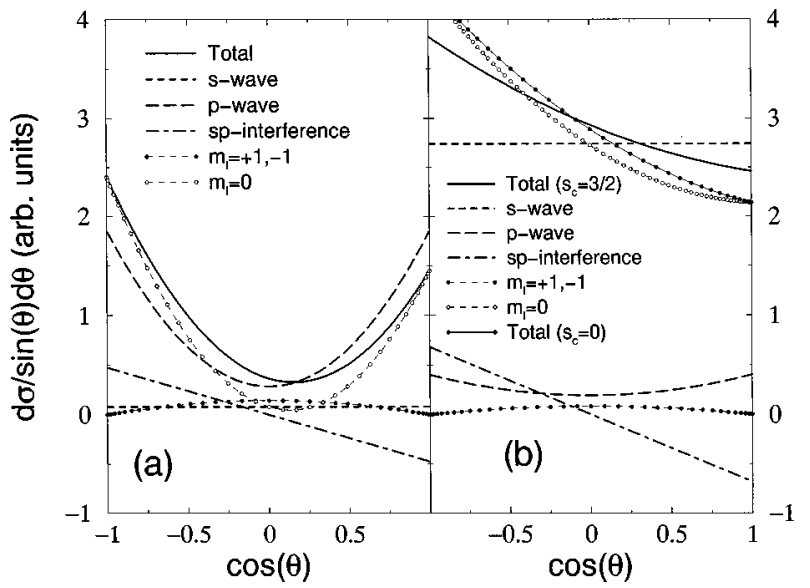

FIG. 2. The absorption contribution, computed in the projectile center of mass, from the spectator-neutron $(\approx 70 \%)$ to the neutroncore (spectators) angular correlation after fragmentation of ${ }^{6} \mathrm{He}$ (left) and ${ }^{11} \mathrm{Li}$ (right) on ${ }^{12} \mathrm{C}$, at $240 \mathrm{MeV} /$ nucleon. The shadowing parameters are $3 \mathrm{fm}$ for ${ }^{6} \mathrm{He}$ and $4 \mathrm{fm}$ for ${ }^{11} \mathrm{Li}$, and equal: $r_{n n}$ $=r_{n \alpha}$. We show the contributions from the neutron-core (spectators) relative $s$ and $p$ waves, their $s p$ interference, and the three different projections on the ${ }^{5} \mathrm{He}$ or ${ }^{10} \mathrm{Li}$ center-of-mass momentum which is very close to the beam direction. To obtain the contributions from the individual projections we assumed zero core spin of ${ }^{9} \mathrm{Li}\left(s_{c}=0\right)$ for ${ }^{11} \mathrm{Li}$. The total result for $s_{c}=3 / 2$ is also shown (right). The curves are shown with the relative normalization obtained from the initial wave function.

$88 \%$ of $p^{2}$ configurations and $12 \%$ of the neutron-core $s^{2}$ configurations for ${ }^{6} \mathrm{He}$, and $20 \%$ of $p^{2}$ and $80 \%$ of $s^{2}$ for ${ }^{11} \mathrm{Li}$. The binding energies and the root mean square radii are $(0.95 \mathrm{MeV}, 2.45 \mathrm{fm})$ and $(0.305 \mathrm{MeV}, 3.34 \mathrm{fm})$, respectively. For the neutron-target interactions we use nonrelativistic optical potentials [8] with the phenomenological neutron- ${ }^{12} \mathrm{C}$ parametrization EDAI-C12 [10] valid for a range of neutron energies from 29 to $1040 \mathrm{MeV}$. We include 35 partial waves in the calculations.

The binding energies and sizes of the initial states and the experimental neutron and core momentum distributions are essentially reproduced with these parameters $[3,4]$. Furthermore, shadowing parameters of 3 to $4 \mathrm{fm}$ maintain or even improve the agreement of these results for ${ }^{6} \mathrm{He}$, and, in addition, the available absolute two-neutron removal cross section is reproduced [5].

Partial wave division. The initial three-body wave function contains in our examples both $s^{2}$ and $p^{2}$ relative neutron-core configurations. Removal of one neutron leaves the remaining neutron-core system (spectators) in a mixture of $s$ and $p$ waves, which after the absolute square and subsequent integration allow diagonal $s$ and $p$ terms as well as an $s p$-interference term. The shapes of the resulting three angular distributions differ substantially and the weighting, which of course is predicted by our model, is decisive. However, the experimentally preferred reaction mechanism might turn out to be different. To gain insight we show in Fig. 2 the contributions from these different partial waves.

The $s$ waves are angle independent and insignificant for ${ }^{6} \mathrm{He}$ and dominating for ${ }^{11} \mathrm{Li}$. The $p$ waves vary symmetrically with angle and the asymmetric $s p$-interference terms change sign for $\cos \theta=0$ and would therefore not contribute 


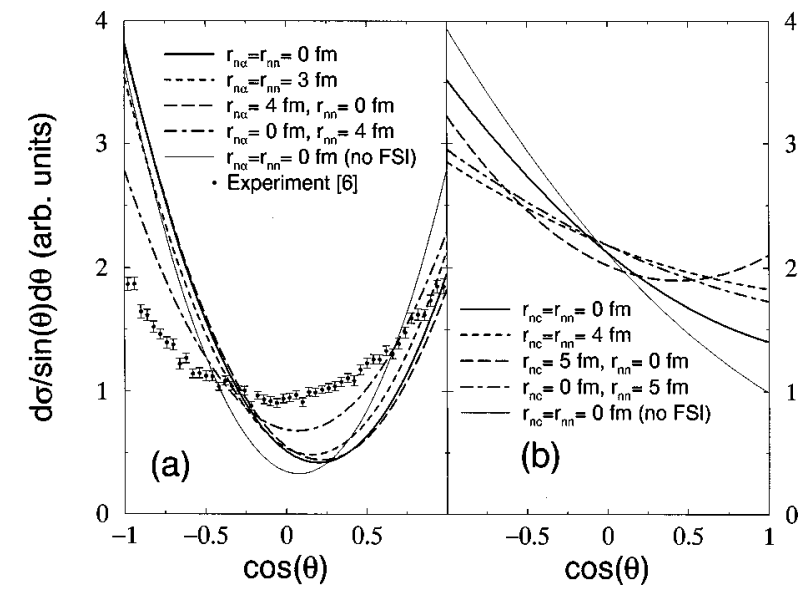

FIG. 3. The total absorption distribution for the same reactions as in Fig. 2. The results for different shadowing parameters are compared with the available measured distributions [6]. For comparison calculations without shadowing and without final state interactions are shown. All calculated curves are normalized to the same value.

to the total cross section obtained by integration over the angle $\theta$. Thus the angular correlation for ${ }^{6} \mathrm{He}$ is essentially determined by the contributions from the $p$ wave and it is therefore symmetric. For ${ }^{11} \mathrm{Li}$ the angular variation is essentially due to the $s p$ interference and therefore very asymmetric.

We also show in Fig. 2 the contributions arising from three different angular momentum projections along the neutron-core (spectators) center-of-mass momentum. For simplicity we assumed zero ${ }^{9} \mathrm{Li}$-core spin $\left(s_{c}=0\right)$ in these computations. The difference between results for $s_{c}=0$ and $s_{c}=3 / 2$ is visible but not substantial. The asymmetry is for both nuclei, again due to the $s p$ interference appearing only for $m_{l}=0$, which contains part of the $p$-wave and all the $s$-wave contributions. These terms with $m_{l}=0$ exhibit a strong angular variation. The terms with $m_{l}= \pm 1$, arising entirely from $p$ waves, are symmetric due to the lack of $s p$ interference and vary relatively little with angle.

Effects of shadowing. We compare in Fig. 3 the measured angular distribution with computations for various shadowing parameters. The first impression is that the computed and measured distributions are quite different. Both the asymmetry and the variation with angle are much larger for the computed curves.

Shadowing is simply removal of unwanted geometric configurations in the initial wave function. We first only remove the part of the three-body wave function within a sphere around the core (long-dashed curves), which implies predominant removal of the relative neutron-core (spectators) $s$ states. We obtain an essentially unchanged distribution for ${ }^{6} \mathrm{He}$, since the $s$ wave only contributes marginally $(\approx 12 \%)$ and all the $p$ waves are reduced by the same amount. The result is a reduction factor independent of angle. For ${ }^{11} \mathrm{Li}$ this shadowing is more visible, since the predominant removal of the dominating $s$ states now produce a more $p$-like structure, i.e., more symmetric but still varying with angle.

If we instead only remove a sphere around the spectatorneutron (dot-dashed curves) we predominantly remove rela-

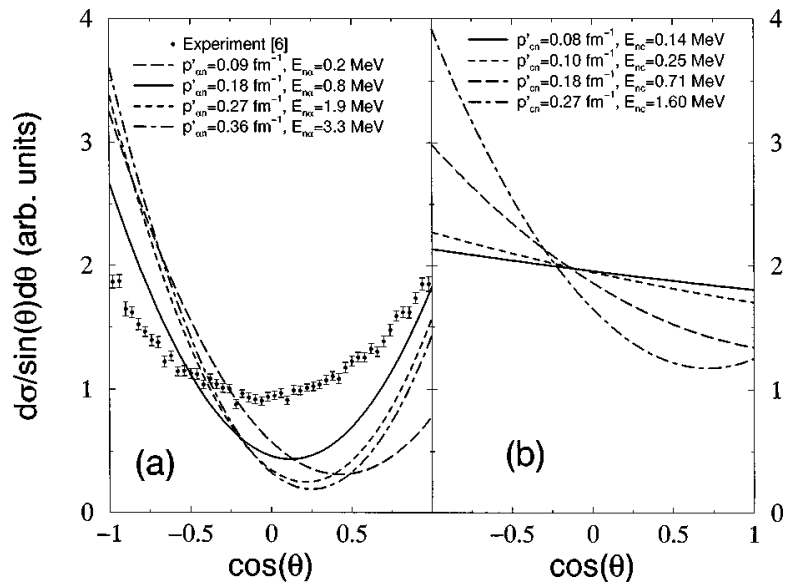

FIG. 4. The total absorption distribution for the parameters and reactions in Fig. 2. The results are given for various relative neutron-core (spectators) excitation energies $E_{n \alpha}$ and $E_{n c}$ $=p_{n c}^{\prime 2} / 2 \mu$. All calculated curves are normalized to the same value.

tive neutron-neutron $s$ states, which must be transformed to the neutron-core Jacobi coordinate system for the spectators. For ${ }^{6} \mathrm{He}$ the removed wave function mostly consists of relative neutron-neutron $s$ waves with one node (hyperspherical quantum number $K=2$ ). The transformed wave function then has a smaller relative content of the dominating $p$ waves and the $p$ and $s p$ contributions decrease relatively compared to the $s$-wave contribution with a smaller asymmetry as the consequence. For ${ }^{11} \mathrm{Li}$ both the removed and transformed wave function are mostly $s$ waves without node $(K=0)$. The reduction of the dominating $s$-wave contribution then produces a more symmetric $p$-like angular distribution.

The final state interaction is essential to reproduce the observed narrow neutron momentum distribution [3]. For the angular correlation the effect is significant but still substantially smaller than the difference to the measured distribution. The final state interaction preserves the total angular integrated contribution from each partial wave. The contribution from the neutron-core (spectators) relative $s$ wave is angle independent. However, including the final state interaction and combining with the $p$-wave contributions the asymmetry for ${ }^{6} \mathrm{He}$ or ${ }^{11} \mathrm{Li}$ increase or decrease, respectively.

Dependence on spectator excitation energy. We have assumed that the reaction is dominated by one-neutron absorption while the remaining neutron-core (spectators) system is undisturbed. The argument is that this high-energy process must be very fast compared to the time scale of the intrinsic motion of the halo particles. The reaction has occurred before the remaining halo particles can change their relative motion and, for example, select an excited state which, in the present cases, must be two-body resonances or virtual states. This is the sudden approximation used so successfully for many other observables $[3,4,11]$. In this picture all continuum states are populated with the probability of occurrence in the initial wave function. However, it is conceivable that a different continuum population arises in these reactions and, in particular, when the beam energy is substantially reduced below the values in the present experiments.

To investigate the sensitivity of the angular correlation to the continuum population we show in Fig. 4 the results when only a state with a given relative neutron-core (spectators) 
excitation energy is populated in the final state. This energy is varied from zero and across the energies of the lowest resonances [11]. We find for ${ }^{6} \mathrm{He}$ that all the distributions vary too much with angle. The lowest energy of $0.2 \mathrm{MeV}$ exhibits a large asymmetry indicating a relatively large admixture of $s$ waves still far from the measured distribution. The higher energies all produce similar distributions even when the energy matches the $p$ resonance at $0.77 \mathrm{MeV}$. The continuum two-body states are essentially $p$ waves due to the low-lying resonance and the distributions in fact resemble the $p$-wave distribution from Fig. 2. Thus, no weighted average of these continuum states can reproduce the measured distribution.

For ${ }^{11} \mathrm{Li}$ the low-lying virtual $s$ states influence the angular distribution. We find a rather flat and asymmetric distribution at low energy, where the $s$ waves dominate completely. As the excitation energy increases the $p$ waves contribute more and more. This produces an increased asymmetry due to the interference term and at higher energies the $p$-wave contribution is clearly pulling the distribution towards symmetry.

Contribution from the participant-neutron. If the participant-neutron is scattered by the target instead of being absorbed it may still be detected and contributes then about $15 \%$ to the measured cross section. The estimate assumes that this neutron arrives within the forward angle where the detection takes place. This relatively small contribution is for technical reasons only estimated approximately. We first approximate the motion of the center of mass of the neutron core (participant-spectator) after the reaction to be in the direction of the beam. Then the participant-neutron and spectator-core relative momentum is approximated, as for an infinitely heavy core, by the momentum of this participantneutron relative to the projectile center of mass. The heavier the core and the higher the beam energy the better the approximations. Finally we obtain the contribution from Eq. (1) by integration over all momenta except the angle between these two momenta. The result is shown in Fig. 5.

The contribution from the participant-neutron is almost symmetric and peaked at $\cos \theta=0$, see the inset of Fig. 5. This behavior is in striking contrast to the contribution from the spectator-neutron which has a minimum at $\cos \theta=0$ for ${ }^{6} \mathrm{He}$ and is rather flat for ${ }^{11} \mathrm{Li}$. The peak corresponds to a preferred direction perpendicular to the beam direction as obtained for forward scattering of a neutron on a target expressed in the rest system of the neutron. Such a distribution is broadened by the intrinsic motion of the scattered participant-neutron with respect to the center of mass of the two spectator particles. Thus, the higher the beam energy and the smaller the halo binding energy, the narrower the distribution.

For ${ }^{6} \mathrm{He}$ the contribution from the participant-neutron only changed the total distribution very little, see Fig. 5. For ${ }^{11} \mathrm{Li}$ a peak appears due to the rapid angular variation of the contribution from the scattered participant neutron compared to the almost constant background from the dominating $s$ waves, see Fig. 5. Before making a direct comparison with measurements it is worth keeping in mind that this result is obtained first as a crude estimate and furthermore with all participant-neutron contributions included independent of possible additional experimental selection.

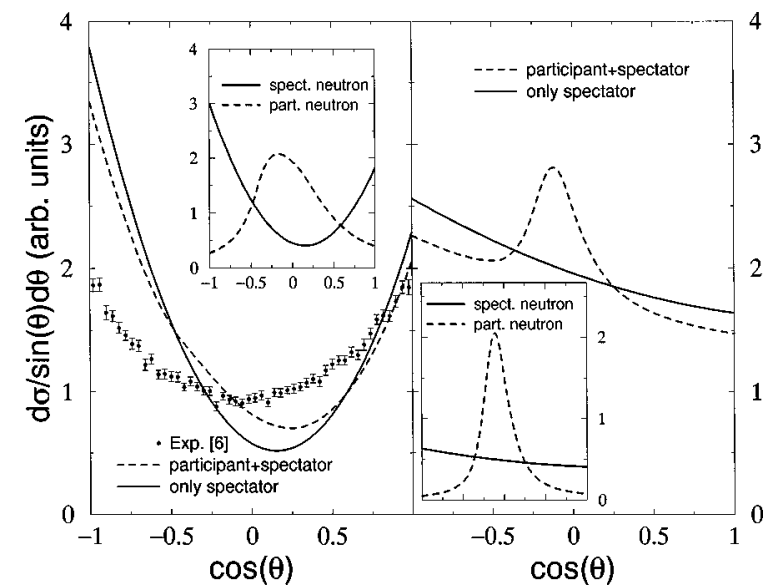

FIG. 5. The angular distribution for the parameters and reactions in Fig. 3. The insets show the two contributions from the scattering process with two neutrons and a core in the final state. Each of these contributions amounts to about $15 \%$, i.e., when the participant (dashed) and the spectator-neutron (solid), respectively, are measured. The solid curves in the external parts give the total contributions when the approximately $15 \%$ from the participant neutron is completely neglected; i.e., we show the sum of the solid curve $(\approx 15 \%)$ from the insets and the total contribution $(\approx 70 \%)$ from Fig. 2. The dashed curves in the external parts show the sum of the solid curves $(\approx 85 \%)$ and the dashed curves $(\approx 15 \%)$ from the inset.

Conclusion. We computed the recently measured angular correlation for breakup reactions of halo nuclei ${ }^{6} \mathrm{He}$ and ${ }^{11} \mathrm{Li}$, i.e., the halo nucleus interacts at high energy with the target and the angular distribution of the relative momentum of the detected ${ }^{5} \mathrm{He}$ and ${ }^{10} \mathrm{Li}$ neutron-core system is measured in a coordinate system with the $z$ axis along the centerof-mass momentum. We used a model which successfully describes essentially all other three-body observables for such systems.

We investigated the contributions from different partial waves of the relative motion of the detected neutron-core system and found constant, symmetric, and asymmetric distributions arising respectively from diagonal $s$, diagonal $p$, and $s p$-interference terms. The zero angular momentum projection on the direction of the ${ }^{5} \mathrm{He}$ and ${ }^{10} \mathrm{Li}$ momenta produces by far the largest variation with angle.

Exciting continuum neutron-core states of a given energy in the reaction with the subsequent decay of these states might be a possible reaction mechanism which however is not supported by the present analysis. The energy is too high to allow time to select specifically for example the two-body resonance states and agreement with the available data is furthermore not improved.

Two processes are possible, i.e., absorption or scattering of one neutron (participant) while the other neutron and the core (spectators) continue undisturbed. The detected neutroncore system may consist of either participant or spectatorneutron and the resulting angular distributions are qualitatively different. The process where the participant-neutron is detected contributes by less than the $15 \%$ predicted by the optical model, since only a narrow cone around the beam direction is selected in the experiment. 
The correlations for ${ }^{6} \mathrm{He}$ and ${ }^{11} \mathrm{Li}$ are qualitatively different, and the computed distribution varies too strongly compared to the data for ${ }^{6} \mathrm{He}$. Provided the experimental correlation is correct this indicates that a subtle reaction mechanism is at work. From the present investigation this can be either a missing constant background, for example, due to suppression of $p$ waves, or a preferred selection of $s$-waves or a relative suppression of zero angular momentum projections.

We thank K. Riisager for continuous discussions and suggestions and T. Aumann and L. Chulkov for a number of clarifying remarks in connection with the experimental data.
[1] P. G. Hansen, A. S. Jensen, and B. Jonson, Annu. Rev. Nucl. Part. Sci. 45, 591 (1995).

[2] M. V. Zhukov, B. V. Danilin, D. V. Fedorov, J. M. Bang, I. J. Thompson, and J. S. Vaagen, Phys. Rep. 231, 151 (1993).

[3] E. Garrido, D. V. Fedorov, and A. S. Jensen, Phys. Rev. C 55, 1327 (1997).

[4] E. Garrido, D. V. Fedorov, and A. S. Jensen, Nucl. Phys. A617, 153 (1997).

[5] E. Garrido, D. V. Fedorov, and A. S. Jensen, Europhys. Lett. 43, 386 (1998).
[6] L. V. Chulkov et al., Phys. Rev. Lett. 79, 201 (1997).

[7] J. Bang and C. A. Pearson, Nucl. Phys. A100, 1 (1967).

[8] J. M. Udías, P. Sarriguren, E. Moya de Guerra, E. Garrido, and J. A. Caballero, Phys. Rev. C 51, 3246 (1995).

[9] G. F. Bertsch, K. Hencken, and H. Esbensen, Phys. Rev. C 57, 1366 (1998).

[10] E. D. Cooper, S. Hama, B. C. Clark, and R. L. Mercer, Phys. Rev. C 47, 297 (1993).

[11] A. Cobis, D. V. Fedorov, and A. S. Jensen, Phys. Rev. Lett. 79, 2411 (1997); Phys. Lett. B 424, 1 (1998). 